\title{
BILATERAL SUDDEN SENSORINEURAL HEARING LOSS IN SCRUB TYPHUS
}

\author{
Dhanashree Ananthashayana Iyengar ${ }^{1}$, Devraj Sharma², Ravinder Singh Minhas ${ }^{3}$, Madhuri Dadwal', Shaweta $^{5}$
}

1 Junior Resident, Department of ENT - HNS, IGMC, Shimla.

2 Professor, Department of ENT - HNS, IGMC, Shimla.

${ }^{3}$ Professor, Department of ENT - HNS, IGMC, Shimla.

${ }_{4}^{4}$ Associate Professor, Department of ENT - HNS, IGMC, Shimla.

5Junior Resident, Department of ENT - HNS, IGMC, Shimla.

ABSTRACT

\section{BACKGROUND}

Scrub typhus, also known as bush typhus is an acute, febrile illness caused by trombiculid mites. The disease is endemic to Southeast Asia and Northern Australia and derives its name from the vegetation that harbours the mite. The causative organism is Orientia tsutsugamushi, a gram negative alpha proteobacterium. The patient usually presents with high-grade fever, an eschar at the inoculation site, generalised lymphadenopathy, organomegaly and pneumonitis. Rarely, the patient may go into acute renal failure, disseminated intravascular coagulation and shock. Sudden sensorineural hearing loss during the course of the disease is a very uncommon presentation and is usually reversible with empirical treatment.

\section{KEYWORDS}

Scrub Typhus, Sudden Sensorineural Hearing Loss, Reversible.

HOW TO CITE THIS ARTICLE: Iyengar DA, Sharma D, Minhas RS, et al. Bilateral sudden sensorineural hearing loss in scrub typhus. J. Evolution Med. Dent. Sci. 2017;6(34):2856-2857, DOI: 10.14260/Jemds/2017/614

\section{BACKGROUND}

Scrub typhus is an acute febrile infection transmitted by the bite of leptotrombidium, chigger mite larvae. ${ }^{1}$ The causative organism is Orientia tsutsugamushi, a gram negative alpha proteobacterium. ${ }^{2}$ It is an obligate intracellular parasite. The organism spreads throughout the body through the haematogenous and lymphatic route with the patient manifesting with a wide variety of signs and symptoms- highgrade fever, myalgia, eschar at the inoculation site, generalised lymphadenopathy and organomegaly. ${ }^{3}$ Some of the complications include pneumonitis, myocarditis, hepatitis and acute renal failure. ${ }^{4}$

Sensorineural hearing loss in scrub typhus is not a common complication. Studies conducted in Sri Lanka have reported sensorineural hearing loss in 19\% of serologically diagnosed cases of scrub typhus.

We report a case of a 47 years old female patient diagnosed with scrub typhus with bilateral sudden sensorineural hearing loss during the course of the disease.

\section{Case Report}

A 47-year-old female patient was referred from a primary health centre in district Mandi of Himachal Pradesh to the emergency department of Indira Gandhi Medical College and Hospital, Shimla with the complaints of fever since 5 days, shortness of breath since 3 days and altered sensorium since 2 days. The fever was high grade and associated with chills and rigors. The patient had shortness of breath at rest. There was irrelevant speech and non-responsiveness to oral commands. She had a history of working in fields but had no

Financial or Other, Competing Interest: None.

Submission 09-02-2017, Peer Review 10-04-2017,

Acceptance 19-04-2017, Published 27-04-2017.

Corresponding Author:

Dr. Dhanashree Ananthashayana Iyengar

Room No. 24, PG Girls Hostel,

IGMC, Shimla-171001.

E-mail:dr.dhanashree@gmail.com

DOI: $10.14260 /$ jemds $/ 2017 / 614$ history of any insect bite. On examination the patient was drowsy, not oriented to time, place and person. Pulse rate was 120 beats per minute and blood pressure was 80/58 mmHg. Systemic examination showed crepitations in bilateral lung fields. Cardiovascular system and per abdomen examination were within normal limits.

The patient was admitted with the Department of Medicine where she was started on intravenous fluids, intravenous ceftriaxone and empirical treatment with doxycycline. The patient subsequently developed respiratory failure and was intubated and put on ventilator support in the Intensive Care Unit. On blood investigations there was neutrophilic leucocytosis, deranged renal function tests and serum electrolytes. Blood and urine cultures showed no organism for the cause of sepsis. Cerebrospinal fluid studies showed neutrophilic leucocytosis. Gene expert for mycobacterium tuberculosis was negative. Since the area is endemic for scrub typhus, Enzyme Linked Immunosorbent Assay was performed on the serum for IgM antibodies for Orientia tsutsugamushi which came out to be positive. The patient was started on empirical Doxycycline (through Ryle's tube) and intravenous ceftriaxone. After three days of endotracheal intubation, elective tracheostomy was performed for better pulmonary toileting on ventilatory support. The patient's vital parameters gradually improved and after 6 days of being on mechanical ventilation the patient was weaned off the ventilator. After confirming that the patient was maintaining saturation at room air, the patient was decannulated and the tracheal stoma was closed.

However, post extubation the patient complained of decreased hearing bilaterally. There was no history of hearing impairment prior to the onset of the symptoms of fever and altered sensorium. The patient had no history of ear discharge or noise trauma. On examination, the tympanic membrane was intact bilaterally. The patient was unable to appreciate tuning fork tests. On pure tone audiometry, the patient was found to have a sensorineural hearing loss bilaterally (Right ear- $56 \mathrm{~dB}$; Left ear- $77 \mathrm{~dB}$ ). Brainstem Evoked Response Audiometry was performed, which showed bilateral prolonged wave morphology and absence of wave in 
in both ears. The hearing loss was found to be $45 \mathrm{~dB}$ on right side and $70 \mathrm{~dB}$ on the left.

The patient was started on systemic corticosteroids, decongestants, vasodilators and oxygen inhalation. The patient showed improvement in symptoms with the subsequent pure tone thresholds showing improvement (Right ear- $34 \mathrm{~dB}$; Left ear- $36 \mathrm{~dB}$ at one month follow-up).

\section{DISCUSSION}

Rickettsial diseases are prevalent throughout the world. Areas such as riverbanks and forest clearings provide optimal conditions for the organism to thrive. The disease is quite prevalent in India, more commonly in the Himalayas, although specific data are not available. ${ }^{5}$

The clinical features of scrub typhus are non-specificfever, eschar at the site of bite, lymphadenopathy, organomegaly. ${ }^{6}$ Complications such as pneumonitis, myocarditis, encephalomeningitis and acute renal failure have been frequently reported. ${ }^{4}$

Hearing loss has been documented as a rare complication of scrub typhus. Bilateral sudden sensorineural hearing loss is an extremely rare presentation.

The mechanism of hearing loss in scrub typhus, though not clear, has been attributed to two possible mechanisms. According to the first mechanism, Rickettsiae directly invade the central nervous system and induce vasculitis in the acute stage and this damages the cochlear division of the VIII cranial nerve. The second mechanism is by vasculitis in the vasa vasorum of the cochlear nerve by a secondary immune mechanism.7-9

Premratna et al report that deafness appears in the second week of the disease. In our patient, hearing loss was reported around the 11th day of the disease. The patient showed improvement in the hearing thresholds with the administration of empirical doxycycline and ceftriaxone along with systemic corticosteroids, vasodilators and oxygen inhalation and is generally reversible.

\section{CONCLUSION}

Bilateral sudden sensorineural hearing loss is a very rare complication of scrub typhus that is generally reversible with empirical treatment.

\section{REFERENCES}

[1] Gavin CKWK, Maude RJ, Paris DH, et al. Diagnosis of scrub typhus. Am J Trop Med Hyg 2010;82(3):368-70.

[2] Ji-In K, Dong-Min K, Lee J. Acute sensorineural hearing loss and severe otalgia due to scrub typhus. BMC Infect Dis 2009;9:173.

[3] Cracco C, Delafosse C, Baril L, et al, Multiple organ failure complicating probable scrub typhus. Clin Infect Dis 2000;31(1):191-2.

[4] Goddard J. Chigger mites and scrub typhus. Infect Med 2000;17:236-9.

[5] Soni K, Chhabra S, Yadav J, et al. Acute reversible hearing loss in scrub typhus. The Internet Journal of Otorhinolaryngology 2012;14(1).

[6] Cowan GO, Cook G. Manson's tropical disease. $16^{\text {th }}$ edn. London, WB Saunders 1996:797-814.

[7] Dolan S, Everett ED, Renner L. Hearing loss in rocky mountain spotted fever. Ann Intern Med 1986;104(2):285.

[8] Walker DH, Parks FM, Betz TG, et al. Histopathology and immunohistologic demonstration of the distribution of rickettsia typhi in fatal murine typhus. Am J Clin Pathol 1989;91(6):720-4.

[9] Mahajan SK, Bakshi D. Acute reversible hearing loss in scrub typhus. J Assoc Physicians India 2007;55:512-4. 